\title{
Systematic review of the epidemiology of non- collision injuries occurring to older people during use of public buses in high income countries
}

- Denise Kendrick ${ }^{a,}$,

- Avril Drummond

- Pip Loganc,

- Jo Barnesd,

- Esme Worthington ${ }^{b}$

- a School of Medicine, Division of Primary Care Faculty of Medicine and Health Sciences, University of Nottingham, Room 1303, Tower Building University Park, Nottingham NG7 2RD, United Kingdom

- ${ }^{b}$ School of Health Sciences Faculty of Medicine \& Health Sciences, University of Nottingham, A floor, Queens Medical Centre, Nottingham NG7 2HA, United Kingdom

- c School of Medicine Division of Rehabilitation and Ageing Faculty of Medicine and Health Sciences, University of Nottingham, Room B108a, Medical School Queen's Medical Centre, Nottingham NG7 2UH, United Kingdom

- ' Transport Safety Research Centre Loughborough Design School, Loughborough University, Leicestershire LE11 3TU, United Kingdom

Received 10 December 2014, Revised 2 June 2015, Accepted 3 June 2015, Available online 7 July 2015

http://dx.doi.org/10.1016/j.jth.2015.06.002

\section{Abstract}

Each year more than 6,000 people are injured on public buses in the UK, approximately half of whom are aged 65 or over. This review synthesises the published literature on the epidemiology of non-collision injuries occurring in older people using public buses, to enable understanding of the size and nature of the problem of injuries, and to explore strategies for improving the safety of public transport for older people.

We searched PubMed, Embase, CINAHL, Web of Science, and Transport International Research Documentation (TRID). Studies were included if they were cross-sectional, case-control or cohort studies. Pairs of reviewers independently screened studies for inclusion, assessed risk of bias, and extracted data. Ten studies were included in the review. Older people and women were found to be over-represented in non-collision injuries. Most injuries occurred during daytime hours and on weekdays. Injuries most commonly occurred whilst passengers were standing and either moving around the bus, boarding, or alighting, and whilst the bus was accelerating or decelerating. Bruising was the most common injury 
amongst emergency department attenders, although between $18 \%$ and $33 \%$ suffered more serious injuries such as fractures or dislocations. Many injuries to older public transport users are potentially preventable. Public transport needs to be safe and accessible, and to be perceived as such by older people to ensure independence in outdoor mobility.

\section{Introduction}

Public transport is often perceived as one of the safest means of transport, since the proportion of casualties that occur on public transport is very low [1]. However, each year more than 6,000 people are injured on buses alone in the UK, with over 400 being killed or seriously injured [2], approximately half of whom are aged 65 and over. Older people may be at increased risk of injury as a result of age-related health conditions such as stroke, arthritis, Parkinson's disease, dementia, sensory or cognitive impairment, balance or mobility problems, and frailty.

Due to the ageing population in the UK [3], most of whom will expect to live many years after retirement in good health, there is an everincreasing number of potential older users of public transport. Older people may also be more reliant than others on public transport due to issues of income, subsidies for public transport, loss of a partner who drove, or loss of a driving licence due to physical or cognitive impairments. Public transport has been found to be vital for older people to provide access to goods and services to enable independent living, to enable older people to contribute to society through working, caring responsibilities or volunteering, and to avoid social isolation, with its attendant negative impact on health [4].

However, people aged 80 years of age and over have been found to make only half the number of outdoor journeys, and to travel less than onequarter of the distance, of those aged 50-54 years [5]. There is also evidence that elderly people find it difficult to utilise transport services because of an inability to carry heavy loads and a fear of crime when outside at night [6]. In addition, older people may be deterred from using public transport if they are afraid of falling [7]. A study of 81 older 
people's experiences of outdoor mobility [8] found that the barriers and enablers to using public transport are a complex mix of environmental, health, societal and psychological factors. A key message of the latter study was that improving independence in outdoor mobility is important in maintaining older people's wellbeing. Clearly, in order to ensure this independence, public transport needs to be safe and accessible, and also needs to be perceived as such by older people.

The aim of this systematic review was to synthesise the published literature on the epidemiology of non-collision injuries occurring in older people during their use of public buses, to enable understanding of the size and nature of the problem of injuries, and to explore strategies for improving the safety of public transport for older people.

\section{Methods}

We undertook a systematic review to critically assess and evaluate all research studies addressing the epidemiology of non-collision injuries in older people using public transport. The review was systematic in that we used an organised method of locating, assembling, and evaluating the body of literature using a set of specific criteria.[9]We searched PubMed, Embase, CINAHL, Web of Science, and Transport International Research Documentation (TRID), which combines records from the Transportation Research Board's Transportation Research Information Services (TRIS) Database and the OECD Joint Transport Research Centre's International Transport Research Documentation (ITRD) Database, from the date of their inception to July 2012. The search terms used for each database are given in appendix A. We also searched reference lists of included studies.

Studies were eligible for inclusion in the review if they:

- Were cross sectional, case-control, or cohort studies

- Were written in English

- Included people aged 60 years and older (including studies that included participants of all ages)

- Included people living in high income countries as defined by the World Bank [8] 
- Reported passengers travelling on public buses and/or coaches (defined as 17 seats or more), overground trains, or trams, who incurred a non-collision injury whilst boarding, alighting or travelling on the vehicle

Studies reporting only injuries resulting from collision incidents were excluded. Studies reporting injuries resulting from both non-collision and collision incidents, which did not separately report non-collision incidents, were included, and this has been highlighted in the description of their findings.

This paper presents only the results relating to injuries incurred on public buses and coaches. i.e. Excluding studies which reported injuries to passengers travelling only on trains or trams.

\subsection{Study selection}

Titles and abstracts of articles were scanned independently by two reviewers $(A D, P L)$ to identify relevant articles to retrieve in full. Where an article appeared to be potentially eligible based on the title, but no abstract was available, the full article was retrieved. Disagreements between reviewers were resolved by consensus-forming discussions with a third reviewer (DK).

Full articles were independently reviewed for inclusion by pairs of reviewers ( $A D, P L, D K)$ using a standardised data extraction form containing the inclusion criteria (study design, participants, transport type) and details of the outcomes of interest recorded in the study (type of injury, mechanism of injury, risk factors for injury). Reasons for exclusion were recorded. Disagreements between reviewers were resolved by discussions between all three reviewers.

\subsection{Data collection process}

A standard form was designed for data extraction which included measures of injury occurrence, injury mechanisms, and risk and protective factors for injury. Subgroup analyses (e.g. injury occurrence by 
age, gender, etc.) were recorded where these were reported. Data were extracted independently by pairs of reviewers ( $A D, P L, D K)$.

Disagreements between reviewers were resolved by discussions between all three reviewers.

\subsection{Risk of bias in included studies}

A recent systematic review of tools for assessing quality and susceptibility to bias in observational studies in epidemiology identified a number of useful assessment tools [10]. Two of these can be used to assess cohort, case-control and cross sectional studies $[11,12]$, and both cover the three domains considered by their authors as fundamental in terms of assessing risk of bias: appropriate selection of participants, appropriate measurement of variables, and appropriate control of confounding.

The risk of bias in included studies was assessed independently by pairs of reviewers $(A D, P L, D K)$ using the Fowkes and Fulton tool [12], and descriptions of the extent to which a study met the criteria were reported. Disagreements between reviewers were resolved by consensus-forming discussions between all three reviewers.

\subsection{Data synthesis}

A narrative synthesis of data was undertaken. The occurrence of injuries was described using the measures reported by included studies (e.g. incidence rates, proportions, etc., and 95\% CI where these were reported). Injury mechanisms were described using frequencies and percentages. Risk and protective factors for injuries were described using frequencies and percentages and measures of association (e.g. relative risks, odds ratios and their 95\% CI), where these were reported by studies. Findings have been summarised in tabular format (see tables $A$ to C).

\section{Results}

The process of study selection is shown in figure A. A total of 1,669 potentially eligible articles were found from the searches, and a further 11 were identified from reference lists. Fifty of these articles were assessed 
as being suitable for retrieval to obtain more detailed evaluation. Seven of these (14\%) could not be found. Of the 43 articles evaluated for inclusion, 33 were excluded, most commonly because they did not include the types of transport of interest $(n=16)$ or did not report a study design of interest $(n=11)$. A table of excluded studies is available, on request, from the authors.

Thus, 10 studies were included in the review, all of which were cross sectional studies.

\subsection{Characteristics of included studies}

The characteristics of the included studies are shown in table A. All studies were published between 1980 and 2005, with three (30\%) being published since 2000 . Five (50\%) were from the UK, one $(10 \%)$ from the USA, two (20\%) from Israel, and two (20\%) from Denmark. Eight (80\%) reported injuries to bus passengers only, and two (20\%) reported injuries to bus and coach passengers. The number of participants ranged from 30 to 9,100 .

Four of the studies reported only non-collision incidents, or reported noncollision incidents separately. The remaining six studies reported injuries occurring in non-collision incidents. These comprised between 8\% [13] and $94 \%$ [14] of all incidents included in the studies. In one study the proportion of non-collision incidents was unclear [15], although, from descriptions of the passenger action at the time of injury, at least some injuries occurred in non-collision events. All studies reported either the mechanism of injury or passenger action at the time of injury, and all studies reported some risk factors for injury, most commonly age and sex.

The findings from the assessment of risk of bias are shown in table $B$. The study design was judged to be appropriate for all studies. In several emergency department (ED) studies it was unclear if the study sample comprised all ED attenders or whether some attenders chose not to participate, and if so, how many non-participants there were and how their characteristics compared with those participating in the study. One 
study excluded 'unreliable and questionable accident reports' [13], but the judgements on which this was based and numbers not included in the study as a result of this were unclear. The extent of missing data appeared to be small in most studies.

The generalisability of most studies was limited, either by data being collected only in one or two EDs, by single bus operators, or by the use of national data restricted to incidents reported to the police. In addition, studies of ED attenders will fail to capture non-medically attended injuries, and those studies relying on self-reported injuries will be subject to some degree of under-reporting. Therefore the scale of the problem of injuries to older public transport users will be underestimated in our review. One study reported on only a proportion of injuries, and many of the studies did not describe their data collection in enough detail to allow an assessment of the quality control procedures and the reproducibility of their data. The propensity to self-report injuries may change over time, hence caution should be taken when interpreting within-study comparisons over time for self-reported injuries. Legislative changes or changes in vehicle design over time may also mean that conclusions and recommendations from older studies may no longer be relevant.

\subsection{Findings of studies}

The findings from each study are summarised in table $\mathrm{C}$.

\subsubsection{Age and sex of passengers}

None of the studies specifically recruited older adults. Two studies recruited participants of all ages but did not report on age of participants $[13,14]$. Four studies reported the age, ranging from 3-89 years [15], 281 years [16], 3-88 years [17] and 13-91 years [18]. Two studies reported mean ages of passengers, which were 56.8 years [16] and 55.6 years [18]. Two reported median ages, which were 58 years (interquartile range (IQR) 6-88) for women and 28 years (IQR 3-84) for men [17], and 60 years for both sexes combined [18]. Age group was reported in three studies, including $56 \%$ aged 55 years or older and $20 \%$ aged 75 years and older [15], 36\% aged over 60 years [20], and $60 \%$ aged over 60 
years [19]. Two studies reported that most serious or fatal injuries occurred between 10-14 years of age for males and $70-85+$ years for females $[20,21]$. One study reported that injured women were significantly older than injured men $(p<0.01)[17]$, and one reported that those aged $\geq 60$ years were significantly more likely to be involved in noncollision events than collision events ( $p$ value not reported) [22].

Two studies did not report participant sex $[13,14,23]$. All other studies reported a higher proportion of female than male participants [15-22], ranging from $67 \%$ [17] to $81 \%$ [19] female. One study reported this difference to be statistically significant $(p<0.01)[17]$.

\subsubsection{Time and day of injury}

Five studies reported time of day or day of week of injuries. [13, 15, 1719]. The highest number occurred between 06.00 and 19.00 in all studies. Two studies from Israel found that injuries most commonly occurred between 12.00 and 19.00 [15] and at 10.00 [18]. Two Danish studies found that injuries most commonly occurred between 09.00-11.00 and 15.00-17.00 [17], and at midday [19]. One study from the USA found that injuries most commonly occurred at 06.00-8.30 and 15.00-19.00 [13]. One study found that significantly more injuries occurred on weekdays than at weekends $(p<0.01)[19]$, and a second found that the highest number of injuries occurred on Fridays and lower numbers at weekends [17].

\subsubsection{Passenger action at time of injury and injury mechanism}

Seven studies reported passenger action at the time of injury. One study of ED attenders in Israel found that $56 \%$ of passengers were standing, $25 \%$ were moving around the bus and $19 \%$ were sitting at the time of injury [15]. A study of ED attenders in Denmark found that $46 \%$ of injuries occurred whilst riding on the bus, $24 \%$ whilst boarding and $29 \%$ whilst alighting, and $68 \%$ of injured passengers had luggage in one or both hands at time of injury [17]. Similar figures were found for a second study of ED attenders in Denmark, with $62 \%$ of injuries occurring whilst riding the bus, $25 \%$ whilst alighting, and $8 \%$ whilst boarding. For injuries 
occurring during riding the bus, $83 \%$ of passengers were standing and $14 \%$ were sitting at the time of injury [19]. In each of these studies, at least $90 \%$ of injuries resulted from non-collision incidents.

Two studies of fatal and serious non-collision injuries reported to the police in the UK between 1994-1998 and 1999-2001 found that the greatest proportion of these injuries occurred whilst passengers were seated, with reported figures of $43 \%$ [20] and 44\% [21]. The proportion of serious non-collision injuries occurring during standing ranged from $28 \%$ [20] to $30 \%$ [21], the proportion occurring during alighting ranged from $17 \%$ [21] to $18 \%$ [20], and the proportion occurring during boarding ranged from $9 \%$ [21] to $11 \%$ [20]. For fatal injuries, between $42 \%$ [20] and $69 \%$ [21] of injuries occurred during either standing, boarding or alighting, with many of these resulting from a fall, slip or trip (figures not reported).

One study of injuries reported by a US bus operator found that the greatest proportion of injuries occurred whilst alighting (36\%), with fewer occurring during boarding (19\%), whilst seated (18\%), and whilst moving around the bus (16\%) [13]. Only $1 \%$ resulted from being caught in doors. However, most (89\%) injuries in this study resulted from collision incidents, and non-collision incidents were not reported separately.

One study of hospital-treated injured passengers on public buses operated by 30 British operators focussed on injuries occurring whilst the bus was moving. Few injuries occurred whilst alighting from (4\%) or boarding (3\%) a moving bus. Most injuries resulted from a fall in the bus (69\%), with a smaller number occurring from falls to the ground (14\%) or door entrapment $(3 \%)$. Those aged $\geq 60$ were significantly more likely to have injuries when boarding buses, in gangways when the bus was moving off, or from door entrapment, than the under 60s. Females were significantly more likely to have gangway injuries than males. There was no significant difference in door entrapment injuries by sex [22]. 
In terms of injury mechanism, six studies reported that acceleration and deceleration were important causes of injury, with one, more detailed, study highlighting that these were more common mechanisms for injuries occurring during riding the bus and on boarding. One study of ED attenders in Israel found that $51 \%$ of injuries occurred on acceleration or deceleration [15], and 9\% occurred when the bus swerved to make a turn. A second study of attenders at an ED in Israel reported that falling whilst standing due to acceleration, deceleration, or sudden turns was the most common injury mechanism (no figures reported) [18].

One study of ED attenders at a UK hospital found that $50 \%$ of injuries occurred on sudden braking whilst waiting to alight, $20 \%$ occurred on moving off quickly after boarding, and 30\% occurred on sudden braking whilst seated [16]. A further study of ED attenders at another UK hospital found that one third of injuries occurred when the bus halted suddenly [14].

A study of ED attenders in Denmark found that $37 \%$ of boarding injuries resulted from acceleration, $40 \%$ from stumbling over steps whilst the bus was stationary, and $23 \%$ from doors closing too early, trapping hands or causing falls. For injuries occurring during riding the bus, $26 \%$ resulted from acceleration, $52 \%$ from deceleration, and $16 \%$ occurred when the velocity was constant. Most (68\%) passengers were standing when these injuries occurred. For injuries occurring during alighting, 47\% were caused by stumbling over steps whilst the bus was stationary, $19 \%$ by the doors closing too soon, $15 \%$ from kerbstones or road works at bus stops or crowding whilst alighting, and 19\% from acceleration [17].

One study of hospital-treated injured passengers on public buses operated by 30 British operators found that $29 \%$ of injuries occurred as a result of an emergency stop or the driver taking other action to prevent an accident. They also reported that $29 \%$ of non-collision injuries occurred when the bus was moving off and $19 \%$ when the bus was stopping or slowing down. The majority (85\%) of the moving-off injuries occurred when moving from a bus stop, and $47 \%$ of these were caused to 
passengers who were still boarding whilst the bus moved off. Accelerating injuries accounted for $23 \%$ of gangway accidents, $83 \%$ of which were caused to passengers moving to their seats. In terms of injuries occurring whilst the bus was slowing down, $37 \%$ occurred on the bus platform, most commonly amongst passengers waiting to alight, and $24 \%$ occurred in gangways, most commonly to passengers moving down the gangway to alight. Staircase injuries represented a large proportion of moving-bus acceleration and deceleration injuries (figures not reported). In terms of injuries occurring whilst the bus was stationary, $96 \%$ of these occurred at bus stops, with $75 \%$ of these injuries occurring on the ground or in the platform area. Seventy per cent of ground accidents occurred whilst boarding (i.e. passengers fell off the bus whilst climbing on). Seventy one per cent of accidents in the platform area occurred whilst alighting (i.e. passengers fell within the bus whilst getting off) [22].

\subsubsection{Injury type, body part injured, severity and outcome of injury}

Four studies reported on the type of injury sustained. Three studies reported injuries sustained by bus passengers attending EDs in the UK [16] and Denmark [17, 19], but used different systems for classifying injuries. At least $90 \%$ of passenger injuries in the two Danish studies occurred in non-collision events, but the proportion of injuries occurring in non-collision events in the UK study was not reported. The UK study reported that bruising was the most common injury (frequency unspecified), and that 33\% of passengers suffered fractures [16]. The first Danish study reported sprains to be the most common injury (51\%), followed by contusions and superficial wounds (23\%), and fractures and dislocations (18\%) [17]. The other Danish study reported contusions to be the most common injury (45\%), followed by fractures (22\%) [19]. None of these three studies compared injury type by age, sex, or other factors.

The fourth study, a national survey of 30 bus operators across the UK, reported injuries occurring in non-collision incidents, but it is unclear if these were self-reported or medically attended [22]. Cuts, grazes or bruises to the head or neck were the most common (29\%), followed by 
cuts, grazes or bruises to the leg or foot (22\%) and cuts, grazes or bruises to the arm or hand (11\%). Fractures to the leg or foot were the most common type of fracture $(3 \%)$, followed by fractures to the arm or hand $(2 \%)$ and fractures to other parts of the body $(0.8 \%)$. Fractures to the head or neck accounted for $0.5 \%$ of injuries. Injury type did not vary by age or sex, except that cuts, grazes or bruises to the leg or foot were commonly reported in those aged $\geq 60$ ( $p$ value not reported). Cuts, grazes and bruises to the head or neck were more common in gangway accidents and when entering or leaving seats. Cuts, grazes and bruises to legs and feet were more common in doorway and platform accidents. Fractures were most often reported for doorway and gangway accidents ( $p$ value not reported) [22].

Five studies reported on the body part injured [14], [15], [17], [19], [22]. One of these studies described only the body part injured for fractures and not for other injuries [19]. The fifth study, reporting data from a national survey of bus operators in the UK, reported body part injured by injury type in non-collision incidents, and has been described above [22].

Two of the three studies reporting body part for all injuries found that limbs were most commonly involved, with the proportion of limb injuries ranging from $33 \%$ [15] to $66 \%$ [17]. The third study, which mainly reported collision incidents [14], reported that injuries to the head and face were most common (47\%), followed by those to the neck $(16 \%)$, and upper and lower limbs (11\% each). One study which only reported the body part injured for fractures found that upper limb fractures were the most common (43\%), followed by lower limb and trunk fractures ( $27 \%$ each), with skull fractures accounting for only $2 \%$ of all fractures [19].

Only one study reported injury severity. This comprised bus passengers attending an ED in Denmark [17] in which $90 \%$ of the injuries occurred in non-collision incidents. Most (61\%) injuries were minor (Abbreviated Injury Scale $(A I S)=1$ ), 23\% were moderate (AIS=2), 13\% were serious $(\mathrm{AIS}=3)$, and $2 \%$ were severe (AIS $=4)$. No significant difference was found in injury severity by passenger action at time of injury. 
Three studies reported on outcome of ED attendance. The first from Denmark, with non-collision injuries comprising 94\% of all injuries [19], found that most (70\%) attenders required no further treatment, $14 \%$ were admitted to hospital (of which, one patient died), 13\% were referred to out-patient follow up, and 3\% were referred for GP follow up. The second study, involving non-collision incidents occurring in Israel, reported that $92 \%$ were discharged home, $6 \%$ were admitted to hospital and $2 \%$ (soldiers) were sent for observation at a military clinic [18]. A third study, from the UK, reported that $13 \%$ of participants were admitted to hospital [16].

\section{Discussion}

\subsection{Main findings}

Our review has found only a small amount of published literature on noncollision injuries occurring among older people on public buses and coaches in high income countries. These findings must therefore be interpreted with caution.

In terms of non-collision injuries, older people and women were found to be over-represented in these types of injuries. Most injuries occurred during daytime hours and on weekdays. They most commonly occurred whilst passengers were standing and either moving around the bus, boarding, or alighting, and whilst the bus was accelerating or decelerating. The data provide some evidence that older people are more likely to have injuries boarding buses, in gangways, and from door entrapment than younger people. There is also some evidence that women are more likely to have gangway injuries than men, that older men are more likely to have staircase injuries than older women, and that older women are more likely to have staircase injuries than younger women. Studies of ED attenders report bruising to be the most common injury, but between $18 \%$ and $33 \%$ suffer fractures and or dislocations, with limbs being most commonly injured. Most injuries resulting in ED attendance are minor, but approximately $40 \%$ are moderate to severe. Most ED attenders are 
discharged home, but between $6 \%$ and $14 \%$ are admitted to hospital and $2 \%$ to $13 \%$ are referred to outpatient clinics. Studies frequently concluded that many injuries to older public transport users were preventable.

\subsection{Strengths and limitations of the review}

This review is the first to focus on passenger injuries sustained by older people using public buses. Although there are methodological problems in examining the literature, our findings demonstrate that these injuries are an important, and underestimated, public health problem.

The most important limitation of this review is that, despite using broad search criteria and including literature from all higher income countries, we found surprisingly few published studies. In addition, many studies were reported in insufficient detail to enable a comprehensive assessment of the risk of bias. We found that the generalisability of most studies was limited and most did not report on data collection in sufficient detail to enable an assessment of the quality of the procedures and the reproducibility of the data. None of the studies included in our review included a comprehensive assessment of injuries occurring on public transport, as that requires data from multiple sources including health service, police, transport operator and self-reported data. Consequently, the scale of the problem of injuries to older users of public buses will be underestimated in our review.

Few studies reported a wide range of participant characteristics or injury details. Some studies reported collision and non-collision injuries combined, and so the specific contribution of non-collision injuries is difficult to assess. The classifications used for injury mechanism, action at time of injury, body part injured and injuries received varied substantially, making it difficult to compare between studies. None of our included studies defined "falls" or what was meant by "boarding" or "alighting". Very few studies reported injury severity. One study (and a subsequent update) specifically reviewed design features that may be associated with bus and coach injuries, but none of our included studies evaluated the impact of design features on injury occurrence. None of the studies 
explored the longer-term impact of injuries on older people, including the deterrent effect of injuries on transport use.

UK legislation in 2000[24] to make buses more accessible for people with disabilities will make access on and off vehicles easier, enabling more vulnerable people to travel on buses. These people may be at greater risk of falls and injuries. In addition, it will reduce the number of seats, and may increase the number of standing passengers, which may increase falls risk. Older buses are not required to comply with new legislation until 2017 [20, 21], and so our findings may not be generalisable beyond this time point. Only one of our included studies [21] reports on injuries since this legislation, but does not report injuries in relation to floor or kerb design. Further research on the impact of this legislation on injury occurrence is required.

\subsection{Comparisons with published literature}

We have found one literature review, published in 2005, with which to compare our findings. This review included literature on bus and coach incidents causing serious injury and death in Europe, published between 1980 and 2004. The review included seven studies and reported findings similar to ours with respect to age, sex and injury mechanism. Women and older people were over-represented in non-collision incidents leading to serious injury or death, and boarding and alighting were common injury mechanisms, as was emergency braking [25].

\subsection{Recommendations for reducing injuries amongst older bus users}

One study (and a subsequent update) reviewed design features that may be associated with bus and coach injuries. The review concluded that slips, trips and falls on vehicles may be caused by slippery floors, weather conditions, uneven floors, unexpected or high stops, steep slopes, lack of visual cues, and physiological changes in older people which affect fall risk, vision, hearing, or memory. Slips, trips and falls whilst boarding or alighting may be caused by the step onto or off the bus being too high, riser steps being of different heights, and passengers carrying objects. 
Falls may be caused by acceleration when the vehicle pulls away before a passenger is seated, deceleration when a passenger is standing while waiting to get off the bus, sharp turns into and out of bus stops, and emergency manoeuvres. Bus stops that are too small physically for stopping require sharp turns to enter or exit the stop. Timetable constraints and congestion can contribute to acceleration injuries, and passengers may feel that they need to stand up before arriving at bus stops to enable them to get off the bus in time, or in case the driver does not stop. Use of single operator buses (i.e. no conductors on board buses) may increase bus driver stress and make it more difficult to keep to schedule. Several design features may lead to injuries when passengers make contact with internal parts of the bus, including unprotected metal grab rails in areas where seated passengers' heads fall forwards and passengers' arms may be struck if they fall against ticket machines, card readers, and rubbish bins with hard edges $[20,21]$.

Two studies included in our review reported being unable to make recommendations about bus design features due to insufficient evidence $[13,22]$. The remaining studies made recommendations for improving bus passenger safety. Four studies recommended restricting numbers of standing passengers, prohibiting standing on buses $[14,17,19]$ or restricting the standing area to the rear or centre of the bus [15]. Three studies recommended less tight schedules in order to make driving less stressful for drivers $[15,19,20]$. Two studies recommended the use of restraints within buses $[14,16]$ and the use, or improved design, of handrails $[14,15]$. One study recommended lowering the level of the bus floor, improving the design of steps, raising the level of bus stops by the use of ramps, repair of roads at bus stops, increased visual control outside the bus, modifications of door closing mechanisms, mechanisms to prevent the bus from starting before passengers have entered or exited the bus, and improving braking systems and shock absorbers [17]. Another recommended minimum measurements for seat widths, seat spacing, spacing of doors, number and height of steps, and floor slopes, and use of textured floors to prevent slips, visual cues for floor obstructions, and minimising hard or sharp protrusions in the bus interior 
[15]. One study recommended ensuring that bell pushes are within easy reach of all seats, providing conductors on busy routes at busy times in order to help passengers, collect fares and deal with unruly passengers, and allow the driver to concentrate on driving, better design of features around the ticket/driver area and near doors to minimise contact injuries, and systems to ensure that drivers are aware of seated passengers wishing to alight [20]. One study recommended an increased number of exclusive bus lanes to prevent some instances of deceleration [19].

\subsection{Implications for research, policy and practice}

Larger studies are required to quantify the size and nature of the problem of non-collision injuries to older people using public buses in higher income countries. As other modes of transport (e.g. trams) are increasingly being used in cities in higher income countries, studies should be representative of the variety of modes of transport used. Studies also need to ascertain the full range of injuries, requiring data from different sources, including primary and secondary care, self-reported injuries from passengers not seeking medical attention, public transport operators, and the police. A systematic method of recording and reporting public transport passenger injuries would enable accurate determination of the scale and nature of this problem, prospective surveillance of injuries, and evaluation of the impact of design, regulatory or legislative changes on injury occurrence. Detailed information needs to be collected on passenger characteristics, injury mechanism, action of the vehicle and passenger at time of injury, vehicle components involved in the injury, injury details, including injury severity and outcome, and the impact of injuries on older people's future travel and use of public transport. Without such data, the problem of non-collision injuries in older people will remain underestimated, opportunities to prevent such injuries will be missed, and their impact on the mobility and well-being of older people will not be acknowledged or addressed.

\section{Conclusions}


Passenger injuries sustained by older people using public buses are an important public health problem which disproportionately affect older women. The incidence of these injuries is likely to be underestimated, since not all injuries are medically attended, and many studies are reliant on self-report. A systematic method of recording and reporting public transport passenger injuries would enable accurate determination of the scale and nature of this problem, prospective surveillance of injuries, and evaluation of the impact of design, regulatory or legislative changes on injury occurrence. Many injuries to older public transport users are likely to be preventable. To ensure older people's independence in outdoor mobility, public transport needs to be safe and accessible, and to be perceived as such by older people. 


\section{References}

1. Department for Transport: Transport Statistics Great Britain 2011.

2. Department for Transport: Reported Road Casualties Great Britain: Annual Report. 2008. London.

3. Cracknell R:

http://www. parliament.uk/documents/commons/lib/research/key_i ssues/Key\%20Issues\%20The\%20ageing\%20population2007.pdf [Accessed 19/7/13]. House of Commons Library Research 2010.

4. Age Concern: http://www.ageuk.org.uk/documents/en-gb/forprofessionals/transport/transport_2008_pro.pdf [Accessed 9/7/13]. Policy Position Papers: Transport 2008.

5. Department of Transport: Older People: Their Transport Needs and Requirements. Department of Transport Publications 2000.

6. Gilhooly M, Hamilton K, O'Neil M, Gow J, Webster J, Pike F: Transport and Ageing: Extending Quality of Life via Public and Private Transport. 2003.

7. Logan PA, Dyas J, Gladman JR: Using an interview study of transport use by people who have had a stroke to inform rehabilitation. Clinical rehabilitation 2004, 18(6):703-708.

8. Marsden G, Cattan M, Jopson A, Woodward J: Older People and Transport: Integrating Transport Planning Tools and User Needs. University of Leeds 2008.

9. U.S. Department of Health and Human Services. Agency for Healthcare Research and Quality: Effective Heath Care Program. Glossary of Terms.

http://effectivehealthcare.ahrq.gov/index.cfm/glossary-ofterms/?termid=70\&pageaction=showterm. [Accessed 27/5/15].

10. Sanderson S, Tatt ID, Higgins JP: Tools for assessing quality and susceptibility to bias in observational studies in epidemiology: a systematic review and annotated bibliography. International Journal of Epidemiology 2007, 36(3):666-676.

11. Health Evidence Bulletins Wales:

http://hebw.cf.ac.uk/projectmethod/appendix8.htm [Accessed 16/7/13]. Questions to assist with the critical appraisal of an observational study eg cohort, case-control, cross-sectional 2004. 
12. Fowkes FG, Fulton PM: Critical appraisal of published research: introductory guidelines. British Medical Journal 1991, 302(6785): 1136-1140.

13. Jovanis PP, Schofer JL, Prevedouros $\mathrm{P}$, Tsunokawa K: Analysis of bus transit accidents: empirical, methodological and policy issues. Transportation Research Record 1991(1322):p. 17-28.

14. Kendall IG, Hassan T, Bodiwala G: A review of injuries sustained by bus passengers. Journal of Accident \& Emergency Medicine 1994, 11(1):57-57.

15. Halpern P, Siebzehner MI, Aladgem D, Sorkine P, Bechar R: Noncollision injuries in public buses: a national survey of a neglected problem. Emergency Medicine Journal 2005, 22(2):108-110.

16. Mabrook AF: Injuries sustained by passengers on buses. Journal of Accident \& Emergency Medicine 1994, 11(3):209-210.

17. Nue Moller B, Grymer F, Christensen ST: Bus accidents. Journal of Traffic Medicine 1982, 10(4):59-62.

18. Bachar R, Alagdem D, Sarov J, Sorkine P, Szold O, Halpern P: Injuries due to falls in urban buses: 100 consecutive cases. Harefuah 1999, 137(77):86.

19. Albrektsen SB, Thomsen JL: A casualty ward analysis of bus passenger accidents. Medicine, Science and the Law 1983, 23(2):102-105.

20. Kirk A, Grant R, Bird R: Bus and coach passenger casualties in non-collision incidents. International Conference: Traffic Safety on Three Continents PTRC Education and Research Services Limited held in Moscow, 2001:14.

21. Kirk A, Grant R, Bird R: Passenger casualties in non-collision incidents on buses and coaches in Great Britain. Proceedings of 18th International Technical Conference on the Enhanced Safety of Vehicles, held Nagoya, Japan, 19-22 May 2003:10.

22. Leyland: Passenger problems on moving buses. 1980 Edited by Transport and Road Research Laboratory. Berkshire, UK: Transport and Road Research Laboratory, .

23. Schneider LW, Klinich KD, Moore JL, MacWilliams JB: Using indepth investigations to identify transportation safety issues for wheelchair-seated occupants of motor vehicles. Medical Engineering \& Physics 2010, 32(3):237-247. 
24. Public Service Vehicles Accessibility Regulations (Disabled Persons): SI 2000/1970.

25. Albertsson $\mathrm{P}$, Falkmer $\mathrm{T}$ : Is there a pattern in European bus and coach incidents? A literature analysis with special focus on injury causation and injury mechanisms. Accident; Analysis and Prevention 2005, 37(2):225-233. 